\title{
Simulated dive in rats lead to acute changes in cerebral blood flow on MRI, but no cerebral injuries to grey or white matter
}

\author{
Marianne B. Havnes • Marius Widerøe • \\ Marte Thuen · Sverre H. Torp • Alf O. Brubakk • \\ Andreas Møllerløkken
}

Received: 27 September 2012/ Accepted: 27 November 2012/Published online: 12 December 2012

(C) The Author(s) 2012. This article is published with open access at Springerlink.com

\begin{abstract}
In this study, the effect of a simulated dive on rat brain was investigated using several magnetic resonance imaging (MRI)-methods and immunohistochemistry. Rats were randomly assigned to a dive- or a control group. The dive group was exposed to a simulated air dive to $600 \mathrm{kPa}$ for $45 \mathrm{~min}$. Pulmonary artery was monitored for vascular gas bubbles by ultrasound. MRI was performed $1 \mathrm{~h}$ after decompression and at one and 2 weeks after the dive with a different combination of MRI sequences at each time point. Two weeks after decompression, rats were sacrificed and brains were prepared for histology. Dived rats had a different time-curve for the dynamic contrastenhanced MRI signal than controls with higher relative signal intensity, a tendency towards longer time to peak and a larger area under the curve for the whole brain on the acute MRI scan. On MRI, 1 and 2 weeks after dive, $\mathrm{T}_{2}$-maps showed no signal abnormalities or morphological changes. However, region of interest based measurements of $T_{2}$ showed higher $T_{2}$ in the brain stem among decompressed animals than controls after one and 2 weeks. Microscopical examination including immunohistochemistry did not reveal apparent structural or cellular injuries in
\end{abstract}

Communicated by Dag Linnarsson.

M. B. Havnes $(\square) \cdot$ M. Widerøe $\cdot$ M. Thuen ·

A. O. Brubakk · A. Møllerløkken

Department of Circulation and Medical Imaging,

Norwegian University of Science and Technology,

7489 Trondheim, Norway

e-mail: marianne.b.havnes@ntnu.no

S. H. Torp

Department of Laboratory Medicine, Children's and Women's

Health, Norwegian University of Science and Technology,

7489 Trondheim, Norway any part of the rat brains. These observations indicate that severe decompression does not seem to cause any structural or cellular injury to the brain tissue of the rat, but may cause circulatory changes in the brain perfusion in the acute phase.

Keywords Diving - Cerebral circulation - MRI · Histology

$\begin{array}{ll}\text { Abbreviations } \\ \text { ADC } & \text { Apparent diffusion coefficient } \\ \text { AUC } & \text { Area under the curve } \\ \text { BBB } & \text { Blood brain barrier } \\ \text { CNS } & \text { Central nervous system } \\ \text { DCE } & \text { Dynamic contrast enhanced } \\ \text { DCS } & \text { Decompression sickness } \\ \text { DTI } & \text { Diffusion tensor imaging } \\ \text { EEG } & \text { Electroencephalography } \\ \text { FA } & \text { Fractional anisotropy } \\ \text { FLASH } & \text { Fast low angle shot } \\ \text { FOV } & \text { Field of view } \\ \text { MD } & \text { Mean diffusivity } \\ \text { MRI } & \text { Magnetic resonance imaging } \\ \text { MTX } & \text { Acquisition matrix } \\ \text { RARE } & \text { Rapid acquisition with relaxation enhancement } \\ \text { RD } & \text { Radial diffusivity } \\ \text { RC } & \text { Relative contrast } \\ \text { ROI } & \text { Region(s) of interest } \\ \text { RSI } & \text { Relative signal intensity } \\ \text { SD } & \text { Standard deviation } \\ T_{1} & \text { Longitudinal relaxation time } \\ T_{2} & \text { Transverse relaxation time } \\ \text { TE } & \text { Echo time } \\ \text { TR } & \text { Repetition time } \\ \text { TTP } & \text { Time to peak } \\ & \end{array}$




\section{Introduction}

Saturation diving is widely used for maintenance and inspection of off-shore subsea systems. Exposure to hyperbaric environments is associated with risk of developing decompression sickness (DCS), arterial gas embolism, neurological symptoms and pulmonary dysfunction (Francis et al. 1990; Mollerlokken et al. 2011; Neuman 2003). Amongst the self-reported symptoms following decompression are conspicuous fatigue, visual disturbances, dizziness, nausea and changes in skin sensitivity (Brubakk et al. 1994). The latter symptoms may be related to the effects of hyperbaria on the central nervous system (CNS).

The long-term health effects of diving have been a matter for discussion for many years, and several studies have been performed to examine the neurological effects of diving (Erdem et al. 2009; Irgens et al. 2007). Saturation divers have reported problems with concentration and memory more frequently than control subjects (Ross et al. 2007; Todnem et al. 1990). In recreational diving accidents, neurological symptoms such as numbness, paraesthesia, dizziness and coordination deficiencies are among the most often reported manifestations of DCS (Vann et al. 2011; Newton et al. 2007).

DCS is a clinical diagnosis associated with a number of different signs and symptoms (Vann et al. 2011). However, in serious DCS neurological symptoms dominate (Moon and Gorman 2003). When neurological damage occurs in divers, the suspected primary cause is vascular gas bubbles. Vascular gas bubbles can enter the arterial circulation by a number of methods, but most commonly due to a right-toleft shunt in the heart, or after barotrauma (Warren et al. 1988). Vascular bubbles can have mechanical, embolic and biochemical effects (Mollerlokken et al. 2011). Entrapment of these bubbles may lead to cellular injury, cerebral edema and increased permeability of the blood-brain barrier (BBB) (Hjelde et al. 2002; Kaakkola et al. 1982). Acute effects can be caused by extravascular bubbles producing pain, or vascular bubbles obstructing and causing strokelike symptoms (Vann et al. 2011). High intensity signals in the white matter on $T_{2}$ weighted magnetic resonance images (MRI) have been seen in divers after dive injuries (Cordes et al. 2000; Hutzelmann et al. 2000), while interestingly, cerebral abnormalities observed in case studies seem to appear at different times-points and are of varying permanence (Jallul et al. 2007). Abnormal electroencephalography (EEG) results have been noted in the temporal or frontal regions after recompression treatment in divers with cerebral DCS (Gronning et al. 2005). The presence of vascular bubbles does not mean that DCS will occur, but the absence of bubbles is considered to be a good indicator of decompression safety (Sawatzky 1991).
Bubbles can also lead to delayed symptom onset in relation to the vascular system. Endothelial dysfunction has been observed after diving in experiments with animals (Nossum et al. 2002) and humans (Brubakk et al. 2005), and is believed to be caused by vascular bubbles. Inflammatory responses have also been investigated in relation to bubble formation and decompression (Bigley et al. 2008). To what extent vascular bubbles and decompression per se affect long-term health, if at all, is still controversial.

In the present study, the effect of a simulated dive on the rat brain was investigated using several MRI-methods, at $1 \mathrm{~h}, 1$ and 2 weeks after decompression. The rat brains also underwent light microscopical examination including immunohistochemistry.

\section{Methods}

Fourteen Sprague-Dawley (Taconic, Denmark) rats weighing $310.75 \pm 17.42 \mathrm{~g}$ were used in the study. All experimental procedures and the care of experimental animals conformed to the European Convention for the protection of vertebrate animals used for experimental and other scientific purposes, and the protocol was approved by the Norwegian Council for Animal Research.

\section{Dive protocol}

Following 1 week of acclimatization, the rats were randomly assigned to one of two groups, diving $(n=9)$ or control $(n=5)$. The rats in the dive group were exposed to a simulated air dive in a $20 \mathrm{~L}$ hyperbaric chamber with a continuous air supply. They were compressed at a rate of $200 \mathrm{kPa} / \mathrm{min}$ to a pressure of $600 \mathrm{kPa}$, maintained at that pressure for $45 \mathrm{~min}$ and then decompressed to the surface $(100 \mathrm{kPa})$ at a rate of $50 \mathrm{kPa} / \mathrm{min}$. The control group was kept in their housing facilities at $100 \mathrm{kPa}$ (normal ambient pressure), breathing air.

\section{Ultrasound}

Immediately after surfacing, the rats were anaesthetized ( $1 \%$ isoflurane mixed with medical air), and the pulmonary artery was monitored for vascular gas bubbles for 30 min by transthoracic echocardiography using a $35 \mathrm{MHz}$ probe (Vevo770, Visual Sonics, Toronto, ON, Canada). Bubbles were identified on the monitor as bright spots in the pulmonary artery and verified with Doppler. The amount of bubbles in the pulmonary artery was graded on a 0-5 scale according to a previously described method (Eftedal and Brubakk 1997), where bubble grade $0=$ no bubbles, $1=$ occasional bubbles, $2=$ at least one bubble/ 4 th heart cycle, $3=$ at least one bubble/heart cycle, 
$4=$ continuous bubbling and $5=$ massive bubbling, also described as "white-out" as individual bubbles cannot be seen. The animals were observed for signs of neurological decompression sickness, such as walking difficulties or paresis, before and after the MRI.

\section{Magnetic resonance imaging}

MRI was performed $1 \mathrm{~h}$ after decompression (acute MRI) and at 1 and 2 weeks after the dive, with a different combination of MRI sequences at each time point. All MRI was performed using a 7 Tesla magnet (Biospec 70/20 AS, Bruker Biospin MRI, Ettlingen, Germany) with watercooled (BGA-12, $400 \mathrm{mT} / \mathrm{m}$ ) gradients. A volume resonator was used for RF transmission, and an actively decoupled rat head surface coil was used for RF reception (Bruker Biospin MRI). During scanning, the anaesthetized (Isoflurane $4 \%$ induction and $2 \%$ maintenance in $30 \% \mathrm{O}_{2}, 70 \% \mathrm{~N}_{2}$ ) rats lay prone in a dedicated water heated rat bed. The head of every animal was fixed in the same position with inbuilt earplugs, tooth bar and nose-mask, to assure the same placement within the magnet from scan to scan.

Acute MRI was performed after the ultrasound, within $1 \mathrm{~h}$ after the dive. Before the MRI acquisition, the rats were anaesthetized and a 25 Gauge neoflon was inserted in the tail vein. After a gradient echo, fast low angle shot (FLASH) pilot scan (acquisition time $1 \mathrm{~min}$ ), a series of $\mathrm{T}_{2}$-weighted images were obtained to visualise anatomical changes and oedema using a turbo spin echo (rapid acquisition with relaxation enhancement, RARE) sequence with RARE-factor $=4$, effective echo time $(\mathrm{TE})=25 / 50 / 75 \mathrm{~ms}$, repetition time $(\mathrm{TR})=4,000 \mathrm{~ms}, 3$ averages, acquisition time $7 \min 12 \mathrm{~s}$. Diffusion weighted images were obtained using an echo planar imaging sequence with 3 directions and 6 b-values $(100 / 200 /$ $400 / 600 / 800 / 1,000 \mathrm{~ms}), \mathrm{TE}=53.47 \mathrm{~ms}, \mathrm{TR}=3,000 \mathrm{~ms}, 6$ averages, acquisition time $8 \mathrm{~min} 24 \mathrm{~s}$. For all of these scans, 17 coronal slices were acquired.

To visualise acute changes in brain perfusion and possible disruption of the BBB, dynamic contrast enhancement (DCE) studies were performed: at first, $\mathrm{a}_{1}$-map was acquired using a RARE sequence with RARE-factor $=4, \mathrm{TE}=7.1 \mathrm{~ms}$, $\mathrm{TR}=341 / 562 / 845 / 1,243 / 1,913 / 5,000 \mathrm{~ms}, 1$ average and acquisition time $7 \mathrm{~min} 55 \mathrm{~s}$. This was followed by a precontrast $\mathrm{T}_{1}$-weighted image (RARE): $\mathrm{TE}=7.0 \mathrm{~ms}$, $\mathrm{TR}=350 \mathrm{~ms}, 8$ averages and acquisition time $1 \mathrm{~min} 41 \mathrm{~s}$. Acquisition matrix (MTX) was $256 \times 144$ zero-filled to $256 \times 192$ giving an isotropic in-plane resolution of $156 \times 156 \mu \mathrm{m}^{2}$. Thereafter, a time-series of $\mathrm{T}_{1}$-weighted images was acquired (RARE-factor $=4, \quad \mathrm{TE}=7.0$, $\mathrm{TR}=300 \mathrm{~ms}$, MTX $128 \times 72$, zero-filled to $128 \times 96,1$ average). With each image taking 5.4 s to acquire, 200 images were acquired over $18 \mathrm{~min}$. After $60 \mathrm{~s}$ of acquisition, a dose of $0.3 \mathrm{mmol} / \mathrm{kg} \quad 0.25 \mathrm{M}$ gadolinium-based contrast agent
(Omniscan, GE Healthcare, United Kingdom) (total volume $\sim 0.36 \mathrm{ml}$ ) was injected intravenously through the neoflon over a period of $5 \mathrm{~s}$. Finally, a post-contrast $\mathrm{T}_{1}$-weighted image was acquired with the same parameters as the precontrast $\mathrm{T}_{1}$-weighted scan. For all acquisitions, the field of view $(\mathrm{FOV})$ was $40 \times 30 \mathrm{~mm}$ and 7 slices á $1 \mathrm{~mm}$ was acquired.

The next day, animals were injected with a single dose of $40 \mathrm{mg} \mathrm{MnCl}_{2}$ (\# 7773-01-5, Sigma-Aldrich Inc., St. Louis, USA) per $\mathrm{kg}$ bodyweight $\left(\sim 318 \mu \mathrm{mol} \mathrm{Mn}{ }^{2+} / \mathrm{kg}\right)$ at a concentration of $100 \mathrm{mM}$ intra-peritoneally to serve as MRI contrast for detecting subsequent inflammation and gliosis 1 week after (Wideroe 2009).

On the follow-up, MRI made seven and 14 days after the dive and $\mathrm{T}_{2}$-weighted images were acquired with the same parameters as described for the acute MRI to study anatomical changes and possible edema associated with tissue pathology. In addition, to evaluate manganese-uptake, a 3D data set was obtained on day seven using a $\mathrm{T}_{1}$-weighted gradient echo FLASH sequence with flip-angle $=30^{\circ}$, $\mathrm{TR}=12 \mathrm{~ms}, \mathrm{TE}=3.25 \mathrm{~ms} . \mathrm{FOV}=30 \times 35 \times 20 \mathrm{~mm}$ and MTX was $192 \times 168 \times 96$ zero-filled to $192 \times$ $224 \times 128$ and the interpolated resolution was $156 \mu \mathrm{m}$ isotropic. Images were averaged 16 times and acquisition time was $52 \mathrm{~min}$ with 16 averages. The transmit field of the volume-coil was considered homogeneous within the FOV, while the spatially inhomogeneous sensitivity of the surface coil used in the 3D $\mathrm{T}_{1}$-weighted FLASH acquisition was corrected for using two additional scans in coupled and single coil operation: $3 \mathrm{D} \mathrm{T}_{1}$-weighted FLASH sequences with the same FOV and contrast parameters as described above but with matrix size $32 \times 32 \times 32$. Acquisition time was two min for each scan. Correction of the high resolution 3D data set was performed using in-house developed software (MATLAB ver. R2010a), and is described in detail elsewhere (Wideroe 2009).

On both 7 and 14 days after the decompression, diffusion tensor imaging (DTI) was performed to evaluate specific white matter injury and to look for changes in white matter. The DTI was acquired with an Echo planar imaging sequence using 30 directions and $b=1,000 \mathrm{~ms}, 5$ images with $b=0 \mathrm{~ms}: \mathrm{FOV}=40 \times 40 \mathrm{~mm}, \mathrm{MTX}=172 \times 172$ giving a resolution of $233 \times 233 \mu \mathrm{m}^{2}$. On day 7 , $\mathrm{TE}=37.5, \quad \mathrm{TR}=3,000 \mathrm{~ms}, 17$ slices á $1 \mathrm{~mm}$ were acquired with two averages giving an acquisition time of $14 \mathrm{~min}$. On day $14 \mathrm{TE}=37.5, \mathrm{TR}=5,000 \mathrm{~ms}, 33$ slices at $0.5 \mathrm{~mm}$ were acquired with six averages giving an acquisition time of $1 \mathrm{~h} 10 \mathrm{~min}$.

\section{MR image analysis}

In-house developed software (MATLAB ver. R2010a, Math Works Inc, Natick MA, USA) was used to calculate 
apparent diffusion coefficient (ADC) maps by fitting a mono-exponential model to the signal intensity of the images with different b-values, while $\mathrm{T}_{2}$-maps were calculated by fitting a mono-exponential model to the signal intensity of the images with different TE-values. The same software was also used to calculate DCE parameters using the average signal intensities within the region of interest specified below. The following DCE parameters were calculated based on the $\mathrm{T}_{1}$-weighted image series acquired during and after gadolinium-based contrast injection: relative signal intensity (RSI) $1.5 \mathrm{~min}$ after contrast injection, $R S I_{1.5 m i n}$, area under the curve (AUC) during the five first min after contrast injection, $A U C_{5 \min }$ and time to peak signal (TTP).

All MR images were visually evaluated with respect to morphological changes and with abnormal signal areas. In addition, Medical Image Processing, Analysis and Visualization software (ver. 5.3.4, Centre for Information Technology (CIT), National Institutes of Health (NIH)) (McAuliffe et al. 2001) were used for regions of interest (ROI) analyses of the image data. ROIs in the frontal, parietal and occipital cortex, hippocampus, putamen, thalamus and brain stem at the level of pons were drawn in the $\mathrm{T}_{2}$-maps (Fig. 1) and copied to the ADC-maps and $\mathrm{T}_{1}$-weighted images to ensure the same placement of ROIs in all image sets. Average voxel values in each of these regions were calculated and compared between groups. The same ROIs were also used to calculate DCE parameters, as described above. For the $\mathrm{T}_{1}$-weighted images used for manganese-enhanced MRI 1 week after the dive, ROI were also drawn in muscle areas lateral to the brain on both sides. Using the average signal intensity from muscle, the mean relative contrast (RC) was calculated in all the other ROI using the formula:

$\overline{R C}_{R O I}=\frac{\overline{S I}_{\text {ROI }}}{\overline{S I}_{\text {muscle }}}$

Where $R C_{R O I}$ is the relative contrast and $S I_{R O I}$ is the mean signal intensity of the region of interest and $S I_{\text {muscle }}$ is the mean signal intensity from the muscle ROI.

DTI analyses were performed with the tools of the FMRIB software library (FSL ver. 4.1.4, Oxford Centre for Functional MRI of the Brain, UK; www.fmrib.ox.ac.uk/fsl). Images were pre-processed to reduce image artefacts due to motion and eddy current distortions by affine transformation and co-registration of the diffusion encoded images to the $b_{0}$ images. Single data sets with severe ghosting artefacts were excluded from further analyses (day 21: $n=6$, day 42: $n=5$ ). Brains segmented out using the Brain Extraction Tool before FDT ver2.0 (both part of FSL) was used to fit a voxel wise diffusion tensor model to the diffusion image data (Behrens et al. 2003). Maps for the fractional anisotropy (FA), mean (MD), radial (RD) and axial diffusivity were created for all animals for days 7 and 14 after decompression. ROI were drawn in the internal capsule, external capsule, hippocampal fimbria, body and splenium of corpus callosum on the FA-maps. Mean FA, $\mathrm{MD}, \mathrm{RD}$ and axial diffusivity were calculated in each ROI in each animal.

\section{Histology}

Two weeks after decompression, the rats were sacrificed with an overdose of pentobarbital $(300 \mathrm{mg} / \mathrm{kg})$ and perfused with $4 \%$ paraformaldehyde in phosphate-buffered saline. Brains were post-fixed in the same fixative and embedded in paraffin, then cut in $8 \mu \mathrm{m}$ thick coronal slices corresponding to -3.25 and $-10.30 \mathrm{~mm}$ from the bregma (Paxinos and Watson 2008) and stained with hematoxylineosin (H\&E) (Cell Path Ltd, UK, Chemiteknikk, Norway and Sigma Aldrich, Germany). The sections were also stained with Luxol fast blue (Chemiteknikk, Norway) for myelin. For immunohistochemistry, the paraffin sections were incubated with antibodies against anti-MAP-2 for neuronal integrity (Sigma, USA), anti-glial fibrillary protein (anti-GFAP) (Millipore, Norway) as a marker for reactive astrocytes, anti-cleaved caspase 3 (Cell Signaling Technology Inc., USA) for apoptotic calls, anti-myelin basic protein (anti-MBP) (Covance, USA) for myelin, antiCD68:FITC (AbD Serotec, Germany) for activated microglia/macrophages and rabbit anti-rat albumin (Nordic, the Netherlands) for BBB leakage. After primary antibody incubation, sections were incubated with rat-antiFITC-biotin (Roche, Basel, Switzerland), horse-antimouse-biotin (Vector Laboratories, Burlingame, CA), goat- anti-rabbit (Vector Laboratories, USA) or Dako Envision+System-HRP (Dako, Denmark). Visualization was performed using a Vectastain ABC kit (Vector Laboratories Inc., USA) and Diaminobenzidine (DAB) kit (Vector Laboratories Inc., USA).

The sections were examined for neuropathology using a Nikon Eclipse 80i light microscope and were analysed by a blinded investigator. To assess any gliosis or vascular proliferation both hemispheres were examined, and the number of GFAP-reactive astrocytes and albumin-labelled vessels were calculated using a $40 \times$ objective with an ocular grid.

\section{Statistics}

PASW Statistics 18 (release 18.0.2, SPSS Inc., Chicago, IL, USA) was used for all statistical analysis and the level of significance was set to 0.05 . Two-sided $t$ tests were used to analyze differences in ADC, $\mathrm{T}_{2}$, DCE parameters, relative contrast in the $\mathrm{T}_{1}$-weighted images, and DTI parameters between groups of decompressed and control animals. 

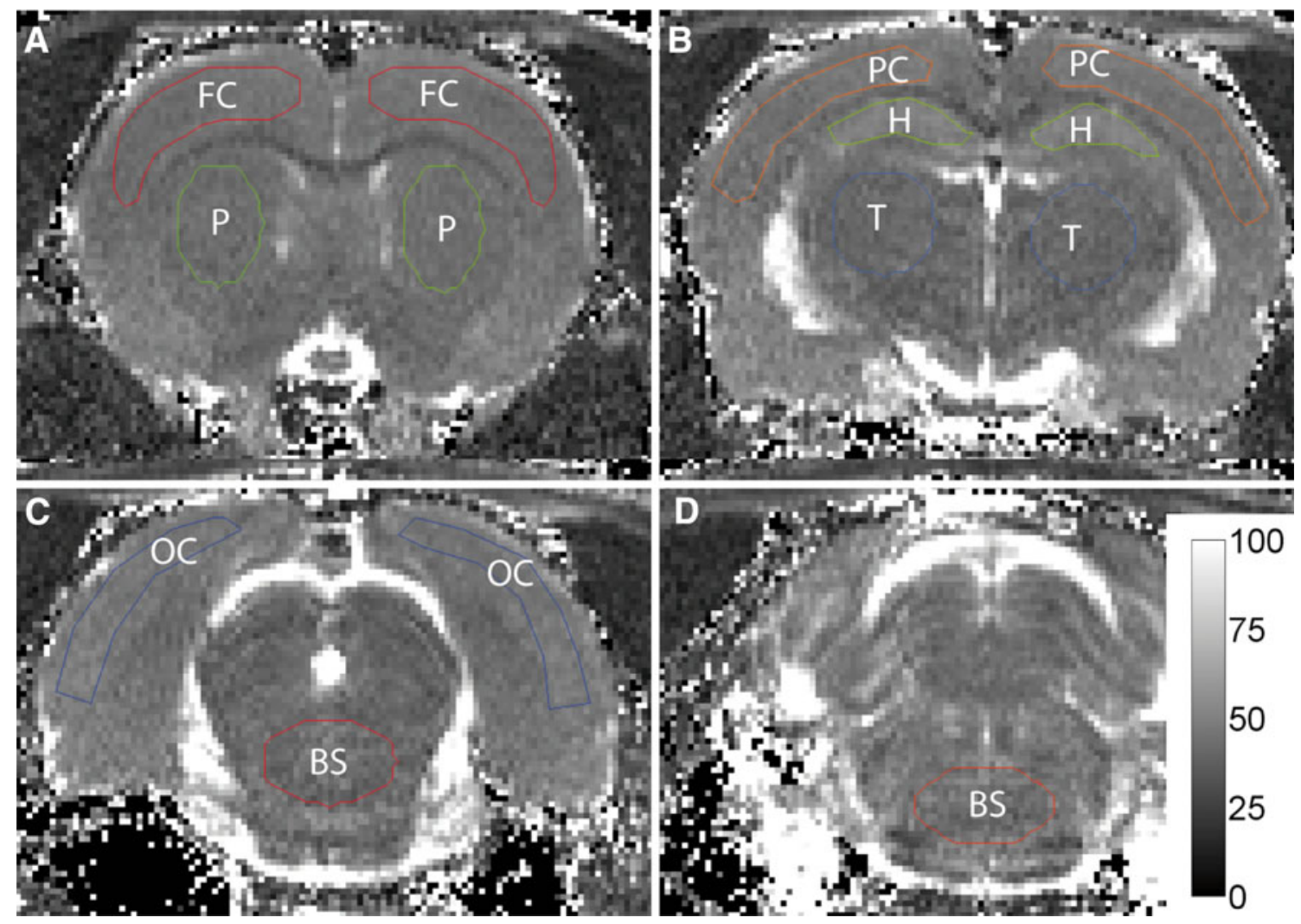

Fig. $1 \mathrm{~T}_{2}$-maps of image slices throughout the brain showing regions of interest used in the analyses. $F C$ frontal cortex, $P$ Putamen, $P C$ Parietal cortex, $H$ Hippocampus, $T$ Thalamus, $O C$ occipital cortex, $B S$ brain stem. Colour bar indicates $\mathrm{T}_{2}$-values (ms)

Histology was analysed by a semi quantitative method by a blinded and trained neuropathologist (SHT).

\section{Results}

Bubble formation and clinical outcome

Of the nine rats in the diving group, three died and six had bubble loads graded between 2 and 4 (median 2) following decompression. The rats that died had considerable bubble loads (visible in tissue after sacrifice), with two dying immediately after the dive and the third showing neurological symptoms of DCS in the form of temporary paralysis of the hind legs; it died after the first MRI scan. The remaining six animals had no clinical symptoms.

\section{Acute MRI findings}

Decompressed rats had a different time-curve for the dynamic contrast-enhanced MR signal than controls, with higher RSI $(P=0.017)$, a tendency towards longer time to peak $(P=0.11)$ and a larger area under the curve for the whole brain $(P=0.099)$ (Fig. 2). Region of interest based analyses of the dynamic contrast-enhanced MRI (DCE

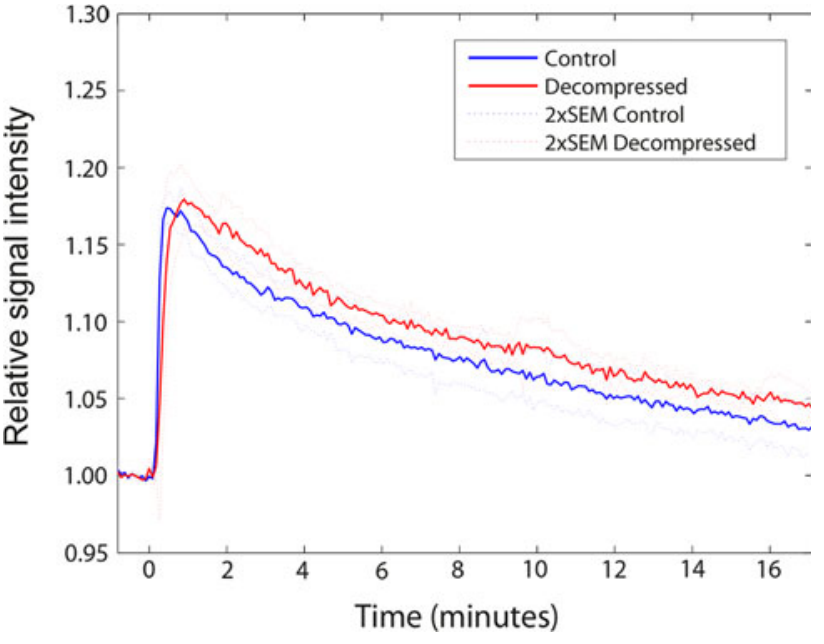

Fig. 2 Plot of the mean relative signal intensity for the whole brain (cerebrum) with time after injection of contrast agent. Blue line represent mean for control animals with $95 \%$ CI (blue dotted line) and red line represent mean for decompressed animals with $95 \% \mathrm{CI}$ (red dotted line)

MRI) data that showed differences between decompressed and control animals in frontal cortex and thalamus. Decompressed animals had higher mean RSI $(P=0.023$, Table 1) and a tendency towards higher TTP and AUC in 
the frontal cortex than control animals $(P=0.076$ and $P=0.068$, respectively). In the thalamus, AUC was higher among decompressed animals $(P=0.033$, Table 1$)$ with a tendency towards higher RSI and TTP $(P=0.068$ and $P=0.104$, respectively). For all other areas, there were no significant differences between the two groups.

Interestingly, the variation in all three parameters (RSI, TTP and AUC) was higher among decompressed animals than controls. There were no specific areas with increased signal in the post-contrast image compared to the precontrast image. The ADC-maps and $\mathrm{T}_{2}$-maps did not show any focal changes indicating pathology in neither the decompressed nor the control animals. Measurements of $\mathrm{ADC}$ and $\mathrm{T}_{2}$ in several brain areas showed no differences between the groups (Tables 2 and 3).

\section{MRI 1 and 2 weeks after decompression}

The $\mathrm{T}_{2}$-maps showed no signal abnormalities or morphological changes 1 or 2 weeks after the decompression. Region of interest based measurements of $\mathrm{T}_{2}$ showed higher $\mathrm{T}_{2}$ in the brain stem among decompressed animals than controls after 1 and 2 weeks, with the same tendency seen in thalamus (Table 3). There was also a similar reduction in $\mathrm{T}_{2}$ in both groups from time 0 to week 1 , with a slight increase to week 2 (Table 3 ) which was related to the administration of manganese on day 1 after decompression. Visual inspection of $\mathrm{T}_{1}$-weighted images after 1 week showed similar manganese-enhancement in both groups, and region based analysis of the images did not show any differences between groups in relative contrast 1 week after administration of $\mathrm{MnCl}_{2}$ (Table 4).
Table 2 Apparent diffusion coefficient (ADC) in different brain areas acutely after decompression

\begin{tabular}{llllll}
\hline & \multicolumn{2}{l}{ Control } & & & Decomp. \\
\cline { 2 - 3 } & Mean & SD & & Mean & SD \\
\hline Frontal cortex & 776.3 & 54.5 & & 795.4 & 67.1 \\
Parietal cortex & 778.8 & 80.5 & & 817.9 & 65.4 \\
Occipital cortex & 826.3 & 71.5 & & 824.6 & 97.2 \\
Hippocampus & 830.0 & 56.4 & & 879.3 & 74.4 \\
Putamen & 761.6 & 38.9 & & 765.4 & 40.3 \\
Thalamus & 783.3 & 47.9 & & 814.2 & 65.4 \\
Brain stem & 856.3 & 80.6 & & 849.0 & 117.8 \\
\hline
\end{tabular}

Mean ADC $\left(10^{-6} \mathrm{~mm}^{2} / \mathrm{s}\right)$ with standard deviation (SD) in groups of control and decompressed rats

No specific white matter abnormalities were seen on DTI and parameters such as fractional anisotropy (FA) (Fig. 3), mean, axial and radial diffusivity were not different between groups in the measured white matter structures (Table 5).

Histology

In routine haematoxylin and eosin $(\mathrm{H} \& \mathrm{E})$ sections and after histochemical and immunohistochemical analyses, no histopathological changes were observed with regard to neuropil, neurons, glial cells, vessels or leptomeninges. In particular, no signs of degeneration of neurons (no red neurons), demyelination, gliosis, inflammation, microglial activation or endothelial injury were detected. Furthermore, no parenchymal immunostaining for albumin was noted consistent with no vascular leakage (Fig. 4).

Table 1 Calculated parameters from the dynamic contrast-enhanced imaging

\begin{tabular}{|c|c|c|c|c|c|c|c|c|c|c|c|c|}
\hline & \multicolumn{4}{|c|}{$\mathrm{RSI}_{1.5 \min }$} & \multicolumn{4}{|c|}{ TTP (min) } & \multicolumn{4}{|c|}{$\mathrm{AUC}_{5 \min }$} \\
\hline & \multicolumn{2}{|c|}{ Control } & \multicolumn{2}{|c|}{ Decomp. } & \multicolumn{2}{|c|}{ Control } & \multicolumn{2}{|c|}{ Decomp. } & \multicolumn{2}{|c|}{ Control } & \multicolumn{2}{|c|}{ Decomp. } \\
\hline & Mean & SD & Mean & SD & Mean & SD & Mean & SD & Mean & SD & Mean & SD \\
\hline Frontal cortex & 1.05 & 0.02 & $1.09 *$ & 0.02 & 0.20 & 0.05 & $0.88^{+}$ & 0.64 & 0.17 & 0.08 & $0.31^{+}$ & 0.11 \\
\hline Parietal cortex & 1.10 & 0.03 & 1.09 & 0.02 & 0.36 & 0.07 & 0.67 & 0.37 & 0.40 & 0.19 & 0.34 & 0.07 \\
\hline Occipital cortex & 1.09 & 0.03 & 1.11 & 0.01 & 0.56 & 0.19 & 0.81 & 0.59 & 0.38 & 0.12 & 0.41 & 0.07 \\
\hline Hippocampus & 1.09 & 0.03 & 1.11 & 0.02 & 0.65 & 0.36 & 1.06 & 0.46 & 0.37 & 0.14 & 0.47 & 0.06 \\
\hline Putamen & 1.08 & 0.01 & 1.09 & 0.02 & 0.54 & 0.26 & $1.17^{+}$ & 0.59 & 0.35 & 0.07 & 0.38 & 0.03 \\
\hline Thalamus & 1.08 & 0.01 & $1.10^{+}$ & 0.02 & 0.43 & 0.28 & 1.62 & 1.27 & 0.35 & 0.03 & $0.44 *$ & 0.06 \\
\hline Brain stem & 1.07 & 0.03 & 1.10 & 0.04 & 0.97 & 0.63 & 2.27 & 1.54 & 0.33 & 0.05 & 0.35 & 0.11 \\
\hline
\end{tabular}

Mean relative signal intensity 1.5 min after injection $\left(\mathrm{RSI}_{1.5 \mathrm{~min}}\right.$ ), time to peak after injection (TTP) in min and area under the curve 5 min from injection $\left(\mathrm{AUC}_{5 \mathrm{~min}}\right)$ with standard deviation $(\mathrm{SD})$ in different brain areas in groups of control and decompressed rats acutely after decompression $P$ values from two-sided $t$ test with unequal variances

Significance is indicated by $* P<0.05, * * P<0.01$

$P<0.1$ is indicated by ${ }^{+}$ 
Table $3 \mathrm{~T}_{2}$ in brain areas at different times after decompression

\begin{tabular}{|c|c|c|c|c|c|c|c|c|c|c|c|c|}
\hline & \multicolumn{3}{|l|}{ Acute } & \multicolumn{5}{|c|}{1 week } & \multicolumn{4}{|c|}{2 weeks } \\
\hline & \multicolumn{2}{|c|}{ Control } & \multirow{2}{*}{$\begin{array}{l}\text { Decomp. } \\
\text { Mean }\end{array}$} & \multicolumn{3}{|c|}{ Control } & \multicolumn{2}{|c|}{ Decomp. } & \multicolumn{2}{|c|}{ Control } & \multicolumn{2}{|c|}{ Decomp. } \\
\hline & Mean & SD & & $\mathrm{SD}$ & Mean & SD & Mean & SD & Mean & $\mathrm{SD}$ & Mean & SD \\
\hline Frontal cortex & 49.5 & 1.1 & 50.1 & 1.5 & 47.1 & 0.6 & 47.5 & 0.9 & 48.1 & 0.6 & 48.3 & 0.9 \\
\hline Parietal cortex & 50.8 & 0.6 & 50.9 & 1.5 & 48.4 & 0.5 & $49.1 *$ & 0.5 & 49.8 & 0.6 & 50.0 & 0.8 \\
\hline Occipital cortex & 52.9 & 0.9 & 52.3 & 1.8 & 50.4 & 1.2 & 51.0 & 0.5 & 52.0 & 0.6 & 52.2 & 0.5 \\
\hline Hippocampus & 55.1 & 0.3 & 55.1 & 1.4 & 51.3 & 0.9 & 51.6 & 1.4 & 53.2 & 0.8 & 52.8 & 1.8 \\
\hline Putamen & 49.2 & 1.4 & 50.5 & 1.4 & 45.1 & 1.3 & 45.4 & 1.5 & 46.6 & 0.6 & 47.2 & 1.0 \\
\hline Thalamus & 48.0 & 1.0 & 48.4 & 1.3 & 43.4 & 0.6 & 44.1 & 0.7 & 44.9 & 0.6 & $45.8 *$ & 0.8 \\
\hline Brain stem & 51.7 & 0.9 & 51.7 & 1.9 & 46.6 & 1.0 & $48.1 *$ & 1.1 & 48.5 & 0.6 & $49.9 * *$ & 0.4 \\
\hline
\end{tabular}

Mean T2 (ms) with standard deviation (SD) in groups of control and decompressed rats. Acutely, 1 and 2 weeks after decompression $P$ values from two-sided $t$ test with unequal variances

Significance is indicated by $* P<0.05, * * P<0.01$

Table 4 Manganese-enhanced MRI

\begin{tabular}{llllll}
\hline & \multicolumn{2}{l}{ Control } & & & Decomp. \\
\cline { 2 - 3 } & Mean & SD & & Mean & SD \\
\hline Frontal cortex & 1.82 & 0.37 & 2.05 & 0.16 \\
Parietal cortex & 1.91 & 0.29 & 2.04 & 0.13 \\
Occipital cortex & 1.75 & 0.12 & 1.73 & 0.11 \\
Hippocampus & 2.10 & 0.28 & 2.04 & 0.16 \\
Putamen & 1.97 & 0.22 & 1.95 & 0.15 \\
Thalamus & 1.93 & 0.18 & 1.82 & 0.18 \\
Brain stem & 1.90 & 0.16 & 1.82 & 0.13 \\
\hline
\end{tabular}

Mean relative contrast with standard deviation $(\mathrm{SD})$ on $\mathrm{T}_{1}$-weighted MRI in different brain areas in groups of control and decompressed rats after 1 week. Increased relative contrast indicated manganeseenhancement

\section{Discussion}

The present study is, to our knowledge, the first controlled, longitudinal study where MRI has been used to evaluate effects of decompression. The results indicate that decompression causes altered brain perfusion in the acute phase, with increased $T_{2}$ in the brain stem on MRI in the 2 weeks after decompression.

To study the ongoing process of the physiological responses to a dive, three time points were chosen for measurement, at $1 \mathrm{~h}, 1$ and 2 weeks after decompression in experimental animals. The dive profile that we used caused bubble formation in all rats, with neurological symptoms (paresis of the hind limbs) noted in one rat within $24 \mathrm{~h}$ and immediate death caused in two rats. The death of three rats indicates the severity of the dive profile. The rats that died had considerable amounts of gas present in their vascular system and tissue. Therefore, death was most probably due to a large number of vascular gas bubbles causing

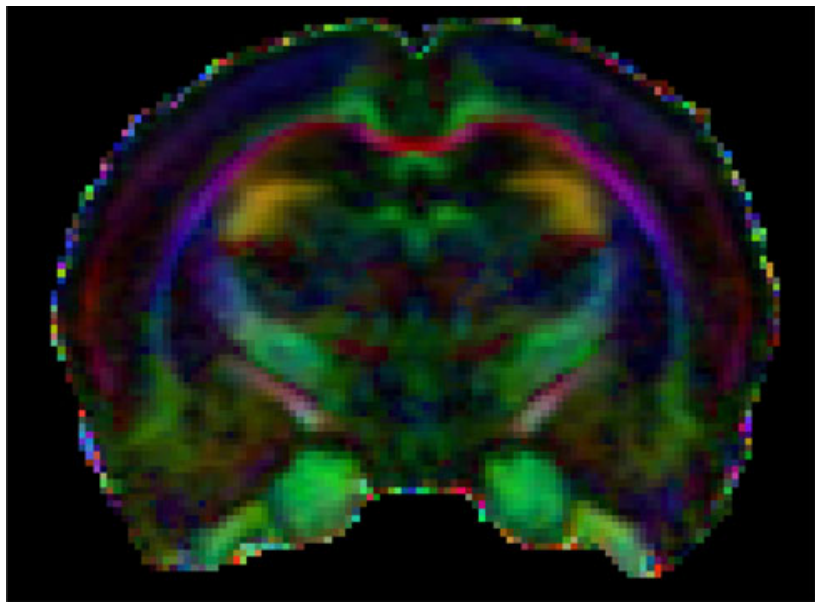

Fig. 3 Image shows representative fractional anisotropy ( $F A$ ) map

cardio-vascular collapse and shock leading to death (Muth and Shank 2000).

The first MRI measurements were designed to look for acute effects in the brain. DCE MRI was performed to assess brain perfusion and permeability of the BBB. The gadolinium-based contrast agent, Omniscan, used in this study remains in the vasculature under normal homeostasis, but is known to leak across the BBB when its integrity is compromised (Wardlaw et al. 2008). The signal change and shape of the signal curve reflects the amount of contrast agent in the brain circulation and any redistribution of the contrast agent from the circulation to the brain tissue. In the present study, the DCE measurements showed that decompressed rats had increased RSI and AUC compared to controls. This indicates an increased blood flow to the brain tissue, and may be explained by an increased oxygen demand secondary to a tissue hypoxia-ischemia caused by the decompression stress. Perfusion changes are previously 
Table 5 Diffusion Tensor Imaging results

\begin{tabular}{|c|c|c|c|c|c|c|c|c|}
\hline & \multicolumn{4}{|c|}{ Week 1} & \multicolumn{4}{|c|}{ Week 2} \\
\hline & \multicolumn{2}{|c|}{ Control } & \multicolumn{2}{|c|}{ Decomp. } & \multicolumn{2}{|c|}{ Control } & \multicolumn{2}{|c|}{ Decomp. } \\
\hline & Mean & SD & Mean & SD & Mean & SD & Mean & SD \\
\hline Corpus callosum body & 0.76 & 0.03 & 0.73 & 0.08 & 0.73 & 0.04 & 0.75 & 0.03 \\
\hline Corpus callosum splenium & 0.79 & 0.03 & 0.73 & 0.08 & 0.69 & 0.04 & 0.67 & 0.08 \\
\hline External capsule & 0.48 & 0.01 & 0.47 & 0.03 & 0.46 & 0.01 & 0.47 & 0.02 \\
\hline Hippocampal fimbria & 0.71 & 0.03 & 0.65 & 0.06 & 0.75 & 0.01 & 0.73 & 0.04 \\
\hline Internal capsule & 0.67 & 0.05 & 0.63 & 0.06 & 0.73 & 0.06 & 0.75 & 0.02 \\
\hline All structures & 0.58 & 0.02 & 0.55 & 0.03 & 0.58 & 0.02 & 0.58 & 0.01 \\
\hline
\end{tabular}

Mean fractional anisotropy (FA) and standard deviation (SD) in different white matter structures in groups of control and decompressed rats after 1 week

$P$ values from two-sided $t$ test with unequal variances

documented in occupational divers in watershed areas of the brain on MRI, and are explained by possible changes in the function of the cerebral microvasculature (Moen et al. 2010). Since DCE was only performed at one time point in our study, the duration of such circulatory changes are uncertain.

As the shape of the signal curves from the DCE in the elimination phase was similar between decompressed and control animals and there were no areas with increased signal intensity in the post-contrast images compared to the pre-contrast images, it was concluded that there was no significant leakage over the $\mathrm{BBB}$ in response to the decompression at the time of imaging in the present study. Decompression has previously been shown to cause increased permeability of the BBB, as illustrated by leakage of dye (Trypan blue) into brain tissue of decompressed rabbits with dye concentration correlating to visible intravascular bubbles (Chryssanthou et al. 1977). We did, however, not find any leakage of albumin in our immunostained brain tissue sections, which would have indicated a BBB leakage. However, using histochemical methods such as Evans blue, increased BBB permeability after decompression has been shown to be temporary and reversible (Nohara and Yusa 1997). In the present study, the acute MRI acquisition started $1 \mathrm{~h}$ after the decompression and the DCE was performed last of all imaging, starting approximately $2 \mathrm{~h}$ after the decompression. Hence, any increased permeability of the BBB may have been reversed at the time of imaging. Future studies should, therefore, aim to perform DCE imaging immediately after the decompression to establish whether this dive protocol causes temporary changes to the BBB permeability. It is important to establish whether there is a change in BBB permeability in response to diving, since this may have consequences for drug administration to divers and compressed air workers. If the BBB is more permeable at a certain time point, then drugs and environmental toxins may penetrate the brain in amounts that could produce toxic or undesirable effects (Chryssanthou et al. 1977). Thus, changes in the integrity of the BBB do not necessarily have any adverse effects per se, but exposure to potentially damaging drugs or environments while it is compromised may do.

Except for the changes in DCE MRI, no focal or general changes indicating cerebral injury were found on anatomical $\left(\mathrm{T}_{1}\right.$ and $\left.\mathrm{T}_{2}\right)$ or diffusion weighted imaging $1 \mathrm{~h}$ after the decompression. The early time point of imaging may explain lack of signal changes on $T_{1}$ and $T_{2}$, but diffusion
Fig. 4 Albumin and GFAP stain from brain stem

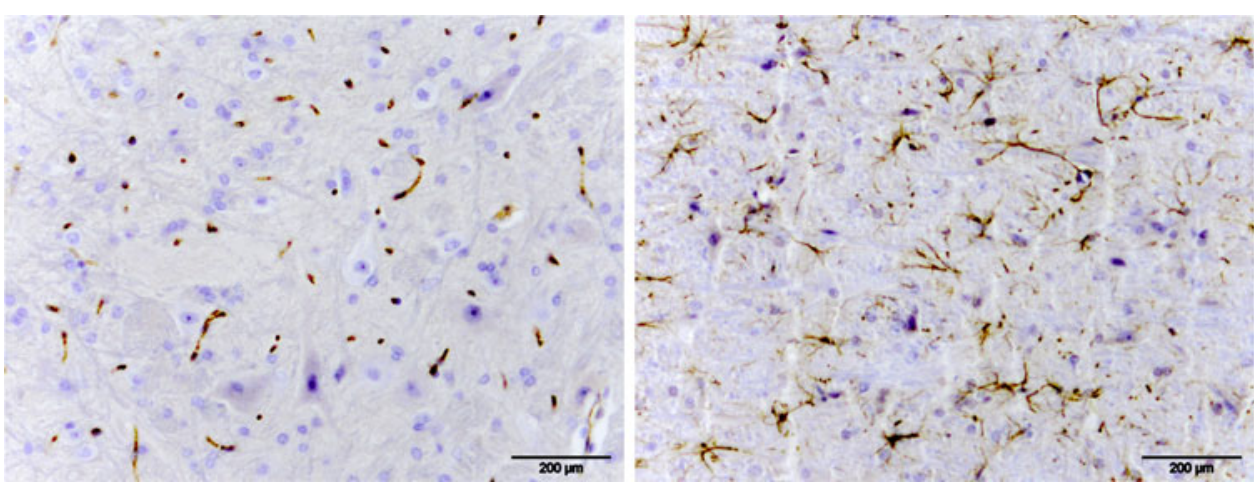


weighted imaging has previously been shown to be a very sensitive method for detecting ischemic lesions (van Everdingen et al. 1998). However, the lack of signs of injury on acute MRI is not surprising, since all but one of the animals showed no neurological signs at that time. In previous studies, focal CNS injuries on conventional MRI were found in the presence of neurological symptoms and signs (Yoshiyama et al. 2007; Jallul et al. 2007; Gronning et al. 2005; Reuter et al. 1997). Moreover, even in the presence of clinical neurological signs suggesting brain involvement, abnormalities on cerebral MRI are uncommon, occurring only in $25 \%$ of the patients in one study (Reuter et al. 1997) and none in another (Gronning et al. 2005).

Hence, lack of detectable injury on acute MRI does not exclude the presence of cerebral injury or that injury can evolve. In fact, on follow-up 1 and 2 weeks after the decompression, changes in $\mathrm{T}_{2}$ were found in brain stem and thalamus among decompressed animals. These delayed findings are in accordance with previously published clinical reports, where MRI abnormalities, especially increased $\mathrm{T}_{2}$ indicating focal CNS injuries, have been found several weeks after the decompression (Reuter et al. 1997; Hierholzer et al. 2000; Jallul et al. 2007). These studies showed MRI abnormalities mostly in the spinal cord, and this is despite the fact that MRIs made during the first $24 \mathrm{~h}$ after onset of symptoms (Jallul et al. 2007) were normal. In the present study, we did not image the spine, but the $T_{2}$ changes were located in the basal parts of the brain. These brain areas are supplied by the basal artery and may have poorer collateral circulation than the neo-cortex, and thus represent more vulnerable areas of the brain in case of large arterial air embolism. The primary cause of increased $\mathrm{T}_{2}$ is increased tissue water content that can be taken as an indication of tissue injury with secondary edema. Reduced capillary density may also lead to increased T2 (Norris 2006). However, histological examinations did not show any signs of tissue injury or vascular changes in the areas with increased $\mathrm{T}_{2}$ or in any other brain areas. The origin of the increased $\mathrm{T}_{2}$ is, therefore, uncertain.

The lack of other findings on MRI after the acute phase could mean that the technique is not sensitive enough to detect subtle tissue changes. However, the MRI results were supported by histological and immunohistochemical examinations that did not show any structural changes in any part of the brain.

One of the hypotheses of this study was that decompression leads to cerebral white matter injuries. However, such injuries may be too subtle to detect on histological examination and anatomical MR imaging, but could be detected using more functional imaging techniques such as DTI where the quantity and directionality of the water diffusion of the tissue can be measured. Injuries to axons and reduced myelination both give specific changes in the quantity and directionality of water diffusion, but no such effects could be detected in our study. Hence, there were no indications that the decompression caused any harmful effects to central cerebral white matter.

There have been several other attempts to study longterm effects in animal models; the CNS has been studied in goats subjected to several (mean 12.5), relatively severe dives over a number of years. In the brains of three out of 36 animals, lesions were seen on MRI $\left(\mathrm{T}_{2}\right)$. However, this was seen in goats with no history of DCS. No evidence of any histopathological damage in the brains of dived animals was found through staining with $\mathrm{H} \& \mathrm{E}$ and glial fibrillary acidic protein (GFAP) (Blogg et al. 2004; Woodger et al. 2001). One suggestion for the lack of significant brain lesions detected in these dived goats was that goats have a slightly different carotid blood supply than humans, with a rete that might act as a filter to some bubbles that pass into the arterial circulation (Daniel et al. 1953).

\section{Conclusions}

In conclusion, the present study indicates that severe decompression does not seem to cause any structural or cellular injury to the brain tissue of the rat, but may cause circulatory changes in the brain perfusion.

Acknowledgments The MRI was performed at the MR Core Facility and the histology was performed at the Cellular and Molecular Imaging Core Facility, Norwegian University of Science and Technology (NTNU). This study has been supported by Statoil, ExxonMobil and Gassco under the Competence Program Diving.

Conflict of interest The authors report no conflict of interest. The authors alone are responsible for the content and writing the paper.

Open Access This article is distributed under the terms of the Creative Commons Attribution License which permits any use, distribution, and reproduction in any medium, provided the original author(s) and the source are credited.

\section{References}

Behrens TE, Woolrich MW, Jenkinson M, Johansen-Berg H, Nunes RG, Clare S, Matthews PM, Brady JM, Smith SM (2003) Characterization and propagation of uncertainty in diffusionweighted MR imaging. Magn Reson Med 50(5):1077-1088

Bigley NJ, Perymon H, Bowman GC, Hull BE, Stills HF, Henderson RA (2008) Inflammatory cytokines and cell adhesion molecules in a rat model of decompression sickness. J Interf Cytokine Res 28(2):55-63

Blogg SL, Loveman GA, Seddon FM, Woodger N, Koch A, Reuter M, Gennser M, White MG (2004) Magnetic resonance imaging and 
neuropathology findings in the goat nervous system following hyperbaric exposures. Eur Neurol 52(1):18-28

Brubakk AO, Bolstad G, Jacobsen G (1994) Helseeffekter av luftdykking. Yrkes- og sportsdykkere (Health effects of air diving. Occupational- and sports divers) (trans: SINTEF), Trondheim

Brubakk AO, Duplancic D, Valic Z, Palada I, Obad A, Bakovic D, Wisloff U, Dujic Z (2005) A single air dive reduces arterial endothelial function in man. J Physiol 566(Pt 3):901-906

Chryssanthou C, Springer M, Lipschitz S (1977) Blood-brain and blood-lung barrier alteration by dysbaric exposure. Undersea Biomed Res 4(2):117-129

Cordes P, Keil R, Bartsch T, Tetzlaff K, Reuter M, Hutzelmann A, Friege L, Meyer T, Bettinghausen E, Deuschl G (2000) Neurologic outcome of controlled compressed-air diving. Neurology 55(11):1743-1745

Daniel PM, Dawes JDK, Prichard MML (1953) Studies of the carotid rete and its associated arteries. Philos T Roy Soc B 237(645): 173-208

Eftedal O, Brubakk AO (1997) Agreement between trained and untrained observers in grading intravascular bubble signals in ultrasonic images. Undersea Hyperb Med 24(4):293-299

Erdem I, Yildiz S, Uzun G, Sonmez G, Senol MG, Mutluoglu M, Mutlu H, Oner B (2009) Cerebral white-matter lesions in asymptomatic military divers. Aviat Space Environ Med 80(1):2-4

Francis TJ, Dutka AJ, Hallenbeck JM (1990) Patophysiology of decompression sickness. In: Bove AA, Davis JC (eds) Diving medicine, vol 2nd. Saunders, Philadelphia, pp 170-187

Gronning M, Risberg J, Skeidsvoll H, Moen G, Aanderud L, Troland K, Sundal E, Thorsen E (2005) Electroencephalography and magnetic resonance imaging in neurological decompression sickness. Undersea Hyperb Med 32(6):397-402

Hierholzer J, Tempka A, Stroszczynski C, Amodio F, Hosten N, Haas J, Felix R (2000) MRI in decompression illness. Neuroradiology 42(5):368-370

Hjelde A, Nossum V, Steinsvik M, Bagstevold JI, Brubakk AO (2002) Evaluation of cerebral gas retention and oedema formation in decompressed rats by using a simple gravimetric method. Scand J Clin Lab Invest 62(4):263-270

Hutzelmann A, Tetzlaff K, Reuter M, Muller-Hulsbeck S, Heller M (2000) Does diving damage the brain? MR control study of divers' central nervous system. Acta Radiol 41(1):18-21

Irgens A, Gronning M, Troland K, Sundal E, Nyland H, Thorsen E (2007) Reduced health-related quality of life in former North Sea divers is associated with decompression sickness. Occup Med (Lond) 57(5):349-354

Jallul S, Osman A, El-Masry W (2007) Cerebro-spinal decompression sickness: report of two cases. Spinal Cord 45(1):116-120

Kaakkola S, Lehtosalo J, Laitinen LA (1982) Changes in blood-brain barrier permeability to drugs in decompressed rats. Undersea Biomed Res 9(3):233-240

McAuliffe MJ, Lalonde FM, McGarry D, Gandler W, Csaky K, Trus BL (2001) Medical image processing, analysis and visualization in clinical research. Comp Med Sy: 381-386

Moen G, Specht K, Taxt T, Sundal E, Groning M, Thorsen E, Troland K, Irgens A, Gruner R (2010) Cerebral diffusion and perfusion deficits in North Sea divers. Acta Radiol 51(9):1050-1058

Mollerlokken A, Gaustad SE, Havnes MB, Gutvik CR, Hjelde A, Wisloff U, Brubakk AO (2011) Venous gas embolism as a predictive tool for improving CNS decompression safety. Eur J Appl Physiol 12:401-409

Moon RE, Gorman DF (2003) Treatment of the decompression disorders. In: Brubakk AO, Neuman TS (eds) Bennett and
Elliott's physiology and medicine of diving. 5th edn. Saunders, pp 600-651

Muth CM, Shank ES (2000) Gas embolism. N Engl J Med 342(7):476-482

Neuman TS (2003) Arterial gas embolism and pulmonary barotrauma. In: Brubakk AO NT (ed) Bennett and Elliott's physiology and medicine of diving. 5th edn. Saunders, pp 557-577

Newton H, Padilla W, Burkart J, Pearl D (2007) Neurological manifestations of decompression illness in recreational diversthe Cozumel experience. Undersea Hyperb Med 34:349-357

Nohara A, Yusa T (1997) Reversibility in blood-brain barrier, microcirculation, and histology in rat brain after decompression. Undersea Hyperb Med 24(1):15-21

Norris DG (2006) Principles of magnetic resonance assessment of brain function. J Magn Reson Imaging 23(6):794-807

Nossum V, Hjelde A, Brubakk AO (2002) Small amounts of venous gas embolism cause delayed impairment of endothelial function and increase polymorphonuclear neutrophil infiltration. Eur $\mathrm{J}$ Appl Physiol 86(3):209-214

Paxinos G, Watson C (2008) The rat brain in stereotaxic coordinates. Academic press, London

Reuter M, Tetzlaff K, Hutzelmann A, Fritsch G, Steffens JC, Bettinghausen E, Heller M (1997) MR imaging of the central nervous system in diving-related decompression illness. Acta Radiol 38(6):940-944

Ross JA, Macdiarmid JI, Osman LM, Watt SJ, Godden DJ, Lawson A (2007) Health status of professional divers and offshore oil industry workers. Occup Med (Lond) 57(4):254-261

Sawatzky K (1991) The relationship between intravscular Dopplerdetected gas bubbles and decompression sickness after bounce diving in humans. York University, Toronto

Todnem K, Nyland H, Kambestad BK, Aarli JA (1990) Influence of occupational diving upon the nervous system: an epidemiological study. Br J Ind Med 47(10):708-714

van Everdingen KJ, van der Grond J, Kappelle LJ, Ramos LMP, Mali WPTM (1998) Diffusion-weighted magnetic resonance imaging in acute stroke. Stroke: J Cereb Circ 29(9):1783-1790

Vann RD, Butler FK, Mitchell SJ, Moon RE (2011) Decompression illness. Lancet 377(9760):153-164

Wardlaw JM, Farrall A, Armitage PA, Carpenter T, Chappell F, Doubal F, Chowdhury D, Cvoro V, Dennis MS (2008) Changes in background blood-brain barrier integrity between lacunar and cortical ischemic stroke subtypes. Stroke: J Cereb Circ 39(4):1327-1332

Warren LP Jr, Djang WT, Moon RE, Camporesi EM, Sallee DS, Anthony DC, Massey EW, Burger PC, Heinz ER (1988) Neuroimaging of scuba diving injuries to the CNS. AJR Am J Roentgenol 151(5):1003-1008

Widerøe M, Olsen Ø, Pedersen TB, Goa PE, Kavelaars A, Heijnen C, Skranes J, Brubakk AM, Brekken C (2009) Manganeseenhanced magnetic resonance imaging of hypoxic-ischemic brain injury in the neonatal rat. Neuroimage 45:880-890

Woodger N, Palmer AC, Blakemore WF (2001) A double blind study of the neuropathology in goats exposed to hyperbaric conditions. Offshore technology report. Department of clinical veterinary medicine, University of Cambridge, Cambridge

Yoshiyama M, Asamoto S, Kobayashi N, Sugiyama H, Doi H, Sakagawa H, Ida M (2007) Spinal cord decompression sickness associated with scuba diving: correlation of immediate and delayed magnetic resonance imaging findings with severity of neurologic impairment-a report on 3 cases. Surg Neurol 67(3):283-287 